\title{
AUTOMATED DETERMINATION OF LATTICE ORIENTATION FROM ELECTRON BACKSCATTERED KIKUCHI DIFFRACTION PATTERNS
}

\author{
STUART I. WRIGHT $\dagger$, JUN-WU ZHAO $\ddagger$ and BRENT L. ADAMS $\dagger$ \\ $\dagger$ Department of Mechanical Engineering, Yale University, New Haven, \\ CT 06520-2157, USA \\ $\ddagger$ Singapore Institute of Standards and Industrial Research, Singapore 0511
}

\begin{abstract}
As described in a previous paper (Wright and Adams, 1990), with the advent of new techniques it has become practical to consider using single orientation measurements for texture investigations.

Advantages of using single orientations measurements over the traditional bulk measurement using pole figures are: direct access to odd- $l$ terms in the series expansion of the Orientation Distribution Function (ODF) (Bunge, 1982) and the capability to measure local textures and to obtain spatial orientation correlation information. The primary obstacle to the use of single orientation measurements is the large investment of direct operator time required to obtain a statistically reliable data set. This study examines a technique that has been developed to automate the orientation identification process. The technique essentially compares an electron backscattered Kikuchi diffraction (BKD) pattern with a set of idealized patterns and finds the best match. The set of idealized patterns is created from a set of orientations which represent a tessellation of the asymmetric region of Euler space. The technique shows promising preliminary results, BKD patterns for silicon and stainless steel were correctly identified by the computer.
\end{abstract}

KEY WORDS Back Scattered Kikuchi diffraction, image analysis, automated orientation determination, stainless steel.

\section{INTRODUCTION}

Currently, the most viable means for measuring local orientations in polycrystalline materials is accomplished by analyzing electron backscattering Kikuchi diffraction (BKD) patterns (Venables and Harland, 1973 and Dingley, 1984). The primary difficulty in automating the measurement of single orientations is indexing the BKD patterns. Current technology requires an operator to determine the proper indexing of the patterns.

The BKD technique is achieved by mounting a polycrystalline sample in a scanning electron microscope so that its surface normal is tilted $70^{\circ}$ away from the incident electron beam. A highly collimated beam is directed onto the sample. Backscattered electrons are diffracted off a small volume of the sample onto a phosphor coated viewing screen which has been mounted in the microscope chamber. The diffracted electrons emit photons upon their collisions with the phosphor screen. A high-gain video camera then transmits this image to a TV screen or directly into a computer. The patterns contain bands which correspond to crystallographic planes in the sample. The intersections of these bands (zone 
axes) denote crystallographic directions. Identifying the Miller indices and location of the zone axes in a BKD pattern allows the orientation of a crystallite to be determined.

\section{THEORETICAL DEVELOPMENT}

The sample is mounted so that its surface horizontal lies parallel to the phosphor screen as shown in Figure 1. The relation between the coordinate frame associated with the sample and that associated with the electron beam is given by:

$$
\begin{aligned}
& \hat{\mathbf{e}}_{1}^{\text {sample }}=-\sin 70^{\circ} \hat{\mathbf{e}}_{2}^{\text {beam }}+\cos 70^{\circ} \hat{\mathbf{e}}_{3}^{\text {beam }} \\
& \hat{\mathbf{e}}_{2}^{\text {sample }}=\hat{\mathbf{e}}_{1}^{\text {beam }} \\
& \hat{\mathbf{e}}_{3}^{\text {sample }}=\cos 70^{\circ} \hat{\mathbf{e}}_{2}^{\text {beam }}+\sin 70^{\circ} \hat{\mathbf{e}}_{3}^{\text {beam }} .
\end{aligned}
$$

The transformation of a vector given in beam coordinates to sample coordinates is given by the following relation.

$$
\mathbf{r}^{\text {sample }}={ }^{s} \mathbf{M}^{b} \mathbf{r}^{\text {beam }}
$$

Where ${ }^{s} \mathbf{M}^{b}$ is the $3 \times 3$ matrix describing the rigid body rotation from the beam reference frame to the sample reference frame and is given by the direction cosines between the orthogonal vectors describing the two coordinate frames. The components of a vector originating at the origin of the beam coordinate frame and extending to the screen are given in terms of the beam reference frame by:

$$
\mathbf{r}=\left(x-x^{*}, y-y^{*}, d\right)
$$

where $x$ and $y$ are the coordinates of the tip of the vector given in screen coordinates, $x^{*}$ and $y^{*}$ are the location of the pattern center in screen coordinates and $d$ is the specimen to screen distance along the $\hat{\mathbf{e}}_{3}^{\text {beam }}$ direction. The pattern center is simply the intersection of the $\hat{\mathbf{e}}_{3}^{\text {beam }}$ direction with the screen, in screen coordinates, as shown in Figure 1.

$$
\left[\left(x-x^{*}\right)+\left(y-y^{*}\right)+d\right]^{-1 / 2}\left[\begin{array}{c}
\left(x-x^{*}\right) \\
\left(y-y^{*}\right) \\
d
\end{array}\right]=\left[{ }^{b} \mathbf{M}^{c}\right]\left[h^{2}+k^{2}+l^{2}\right]^{-1 / 2}\left[\begin{array}{l}
h \\
k \\
l
\end{array}\right]
$$

The transformation matrix ${ }^{b} \mathbf{M}^{c}$ can be obtained by finding the locations of three zone axes on the screen and then solving three simultaneous linear equations. The orientation, $\mathbf{g}$, of the crystal in which the beam is located is then given by:

$$
\mathbf{g}^{T}={ }^{s} \mathbf{M}^{b b} \mathbf{M}^{c} .
$$

The orientation can be uniquely determined using Eq. (5) given the location of three zone axes on the screen and their Miller indices (two are identified by the user and a third determined by geometric considerations), the specimen to screen distance, $d$, and the location of the pattern center. 


\section{two-dimensional diagram of BKD configuration}

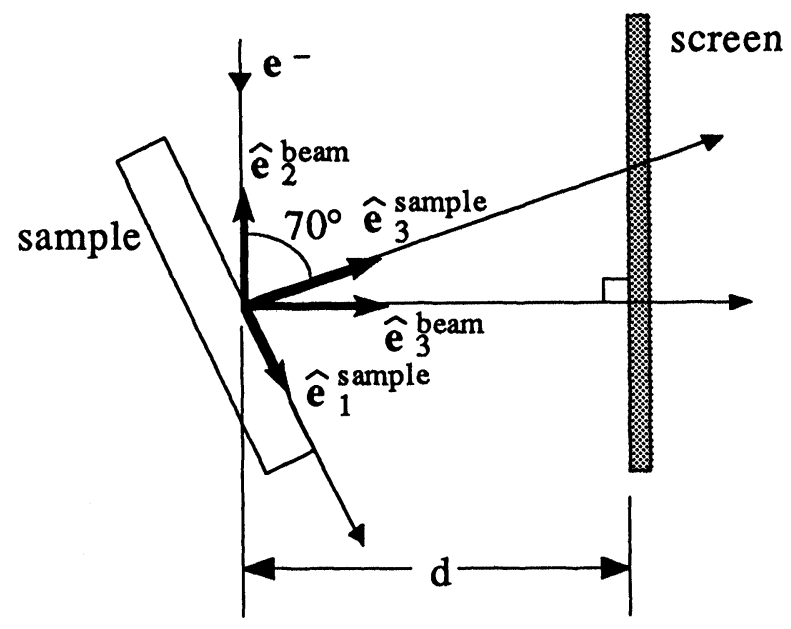

three-dimensional diagram of $\mathrm{BKD}$ configuration

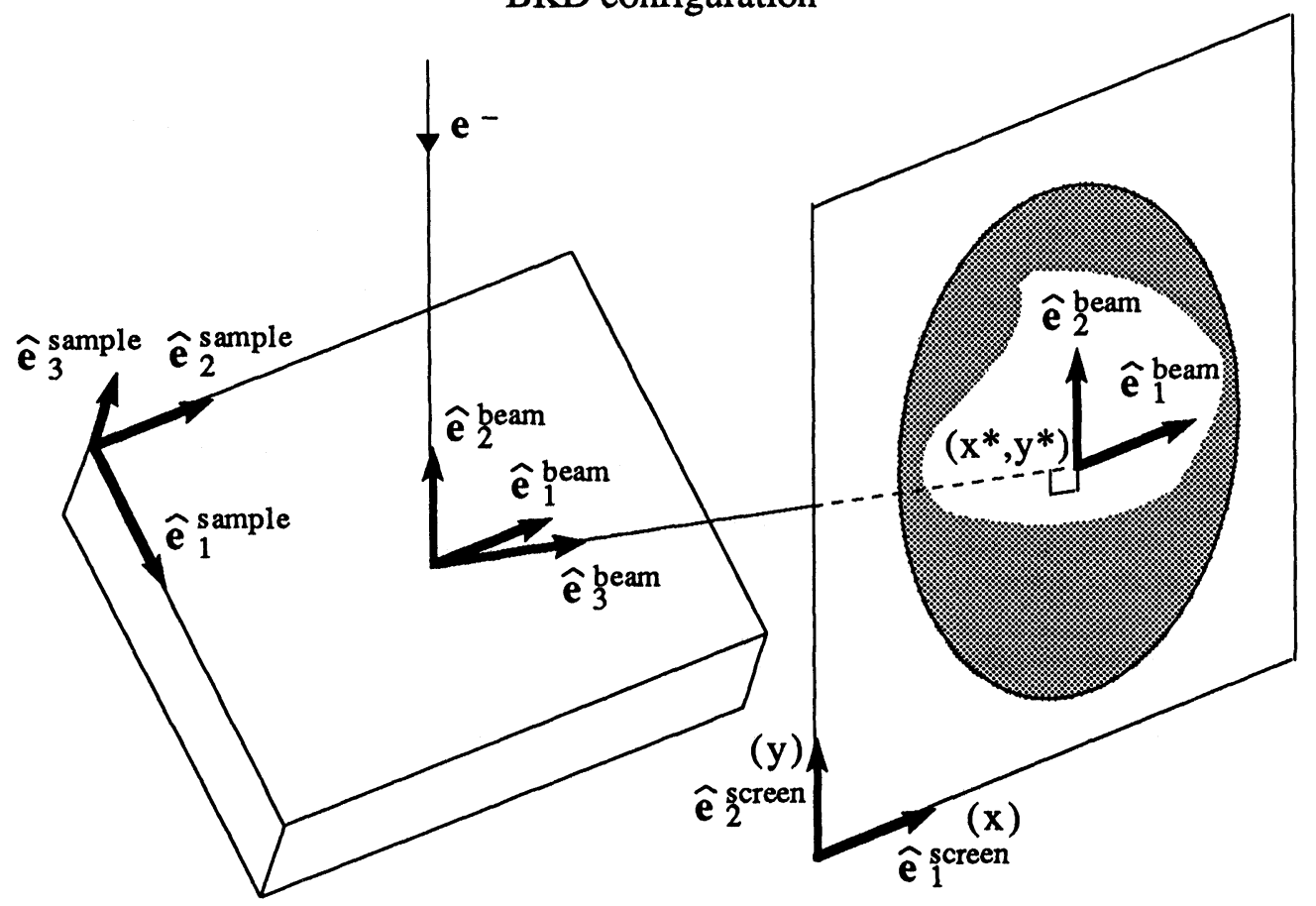

Figure 1 Schematic description of reference frames in BKD system. 


\section{GENERAL PROCEDURE}

In contrast to the current technique of indexing BKD patterns by identifying the location and indices of two zone axes in the patterns; the proposed technique requires the computer to compare the digitized pattern with a set of ideal patterns. These ideal patterns are essentially templates containing a finite set of zone axes. These templates were constructed by tessellating the asymmetric region for cubic crystallographic symmetry of Euler space into $2.5^{\circ} \times 2.5^{\circ} \times 2.5^{\circ}$ cubes. This resulted in 43,488 orientations. The location of a given zone axis in screen coordinates is determined using the following relationship:

$$
\begin{aligned}
& x=x^{*}+\frac{d}{\left(h_{j} h_{j}\right)^{1 / 2}} \frac{M_{1 j} h_{j}}{M_{3 j} h_{j}} \\
& y=y^{*}+\frac{d}{\left(h_{j} h_{j}\right)^{1 / 2}} \frac{M_{2 j} h_{j}}{M_{3 j} h_{j}}
\end{aligned}
$$

where $\mathbf{h}=(h k l),{ }^{b} \mathbf{M}^{c}$ is the rotation matrix as previously defined, $\left(x^{*}, y^{*}\right)$ is the location of the pattern center and $d$ is the specimen to screen distance.

A template was constructed for each tessellar using eight crystallographic poles and their equivalent directions. The poles contained in the set were primary poles in the BKD patterns and were the same for each template. The location at which each pole intersected the plane of the phosphor screen was calculated. Many of the poles intersected the screen plane in positions outside of the region defined by the phosphor screen. These poles were not included in the construction of the template since they would not appear in an experimentally obtained pattern. For the configuration used in this study, an average of approximately fifteen poles lay within the region of the screen in each template.

Each template was placed over the BKD pattern and an average intensity per pole calculated using the following:

$$
\bar{I}^{i}=\frac{1}{n^{i}} \sum_{j}^{n^{i}} I\left(x_{j}^{i}, y_{j}^{i}\right)
$$

where $n^{i}$ represents the number of poles from the global set appearing in the $i$ th template. $x_{j}^{i}, y_{j}^{i}$ represents the location of the $j$ th pole in the $i$ th template in the pattern. $I\left(x_{j}^{i}, y_{j}^{i}\right)$ represents the gray level (black equals zero and white equals $255)$ in the pixel map of the image for a pixel located at $x_{j}^{i}, y_{j}^{i}$ where $x_{j}^{i}$ and $y_{j}^{i}$ are rounded to their nearest respective integers. The template with highest average intensity was then considered to represent the orientation which best fit the pattern to be identified.

Although this procedure has been described for cubic crystal symmetry, the development is easily extended to all crystallographic symmetries.

\section{ADDITIONAL ELEMENTS OF THE PROCEDURE}

The size of the tessellation blocks was selected after automation was attempted using larger sized blocks. The $2.5^{\circ}$ dimension was found to be the largest size that 
still resulted in correct indexing of the patterns. It was noted that the $2.5^{\circ}$ dimension caused the maximum movement of a zone axis between neighboring orientations to be approximately half the size of the spot representing the zone axis in the image. However, this would not be consistent for all microscopic conditions or materials. The size of the bands, which intersect to form zone axes, is dependent upon the voltage of the electron beam and the lattice parameter of the material. A good introductory description of the effects of lattice geometry on the BKD bands is given by Schmidt and Olesen (1989).

To determine a more correct solution a local search through Euler space inside the tessellation cube can be implemented. However, this adds substantial time to completing the identification procedure relative to the time required to search through the templates for the best fit.

The images were also distorted due to lens distortion in the camera. This distortion was corrected by assuming a parabolic foreshortening of the image in the $x$ and $y$ directions. This foreshortening was incorporated into the construction of the templates. The maximum amount of correction was approximately fifteen pixels.

Many of the images exhibited a significant brightness gradient. It was found that this biased the results towards templates having a higher percentage of poles in the bright region. Thus, it is necessary to either remove the brightness gradient from the image or consider intensities in the pixel map relative to their local neighborhood. Both methods were used in this study and gave satisfactory results. One of the problems with the local neighborhood approach is determining the size of the neighborhood. Flattening of the brightness gradient was implemented by finding the average for each column and row of pixels in the image. The column and row averages were then fit to second order curves. The curves were then flattened and the change in the second order curves to the flat curves was used to adjust the image brightness. This was effective in reducing the image brightness gradient. However, some of the contrast between zone axes and the background seemed to have been reduced.

It was found that pixel to pixel intensity variations were large enough to influence the selection of the best fit. One technique found to be effective in reducing the local variations was convolution of the image with a smoothing mask. It was found that a mask of dimension $5 \times 5$ or greater was required. Another method was to simply divide the picture up into $10 \times 10$ pixel blocks and calculate an average intensity per pixel in each of these blocks.

The most important refinement needed to process the image in a way that would lead to a correct indexing was to highlight the zone axes. This was accomplished by convoluting the image with a mask which magnified differences between the zone axes and their surrounding neighborhoods. One such mask is shown in Figure 2.

This represents one quadrant of a convolution mask with a smoothing mask at the center $(a=1 / 9)$ surrounded by zeros everywhere except along a 30 pixel radius circle where $a b(b=-1 / 35)$ is located every $10^{\circ}$ along the circle.

This mask not only increases the zone axis contrast but it also causes the image gradient to disappear and reduces local intensity variations. A mask of this type was found to be necessary in obtaining a correct solution for lower quality images. 


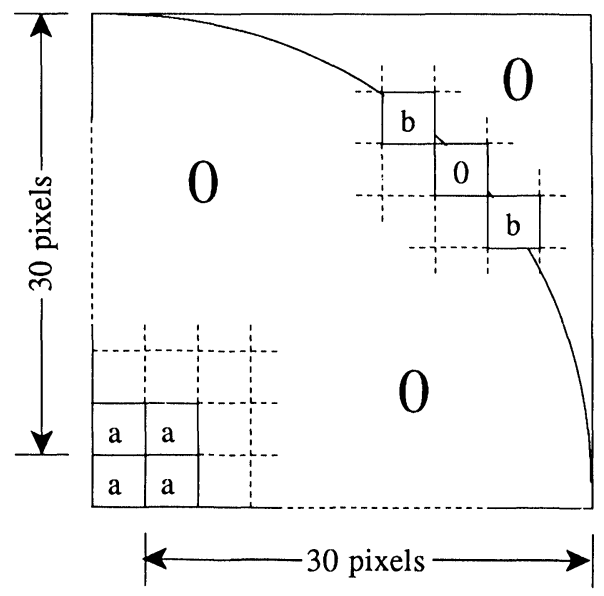

This represents one quadrant of a convolution mask with a smoothing mask at the center $(a=1 / 9)$ surrounded by zeros everywhere except along a 30 pixel radius circle where $a b(b=-1 / 35)$ is located every $10^{\circ}$ along the circle.

Figure 2 Convolution mask for enhancing zone axis contrast.

\section{RESULTS AND SUMMARY}

The algorithm was used on four images: a silicon (fcc) image, two stainless steel (bcc) images and a tungsten (bcc) image. The silicon and tungsten images were obtained by averaging eight video frames and the stainless steel images were single frame images. A correct result was obtained for the silicon image after minimal image processing. The brightness gradient was flattened out and the picture was divided into $10 \times 10$ pixel blocks, which were averaged as previously described. The stainless steel images required more extensive processing. Convolution masks for highlighting the zone axes were passed over the images and a correct solution was obtained (see Figure 3d). The effect of the passes on an original stainless steel image is shown in Figures $3 a, 3 b$ and $3 c$.

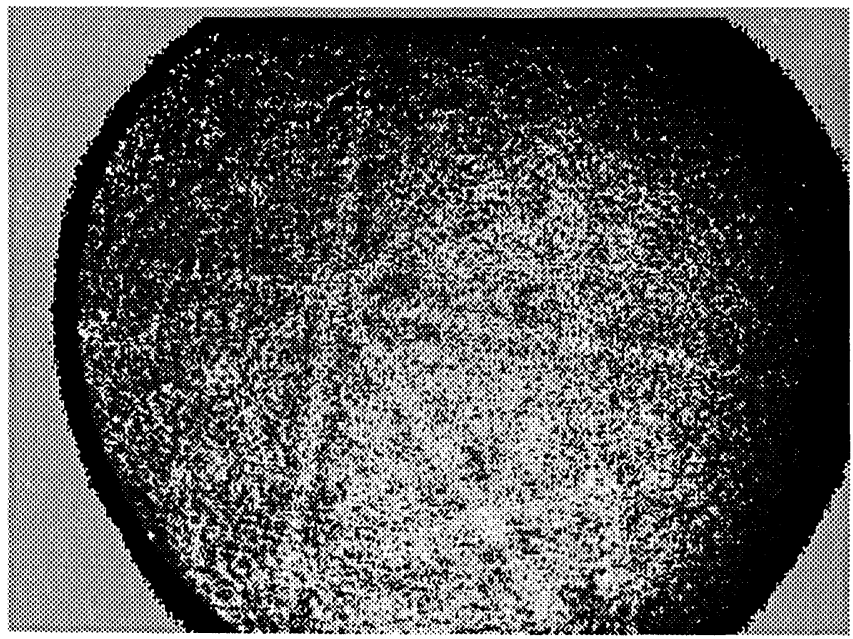

Figure 3a Original digitized image from stainless steel. 


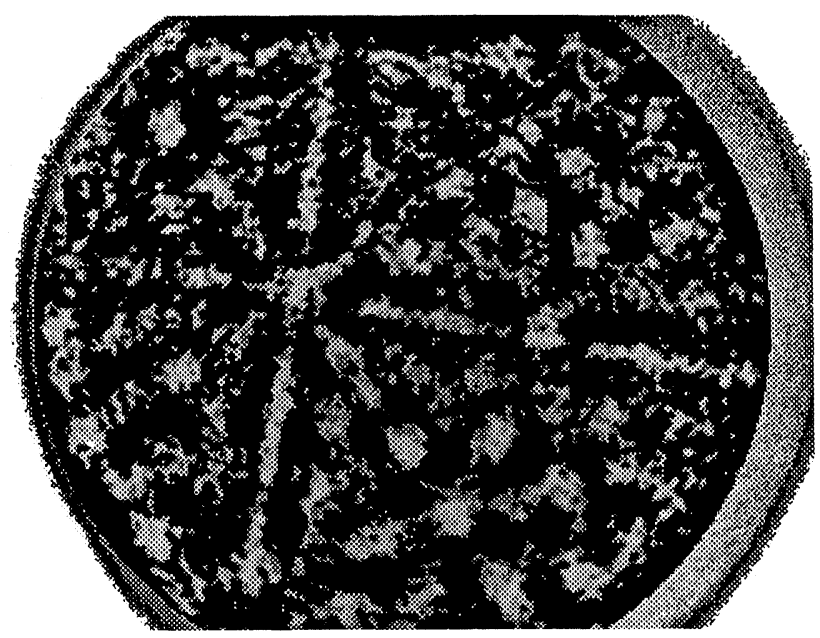

Figure 3b Stainless steel image after one pass of the zone axis enhancement convolution mask.

The attempt to identify the tungsten (bcc) image was not successful. However, the tungsten image was obtained for a larger specimen to screen distance $(d)$ than the silicon and stainless steel images. This resulted in a considerable decrease in the number of poles from the global set appearing in the templates (from an average of fifteen poles to six poles per template). Thus, the templates were not as discriminatory and incorrect solutions were obtained. This result suggests that the closer the phosphor screen can be placed to the sample the more likely the algorithm will select the template representing the correct orientation.

The algorithm was written in MPW Pascal and run on a Macintosh IIx with 5MB RAM. The search through the templates required 26 seconds. The

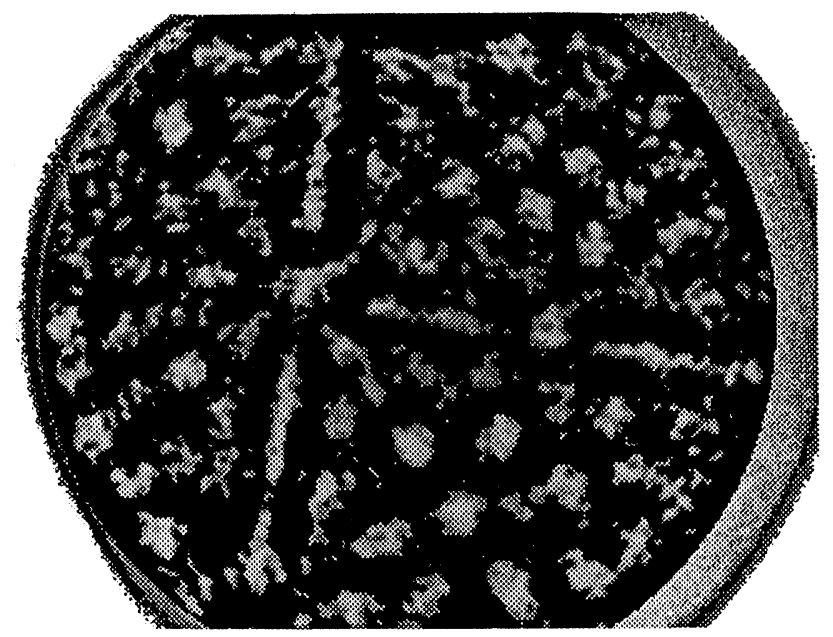

Figure 3c Stainless steel image after two passes of the zone axis enhancement convolution mask. 


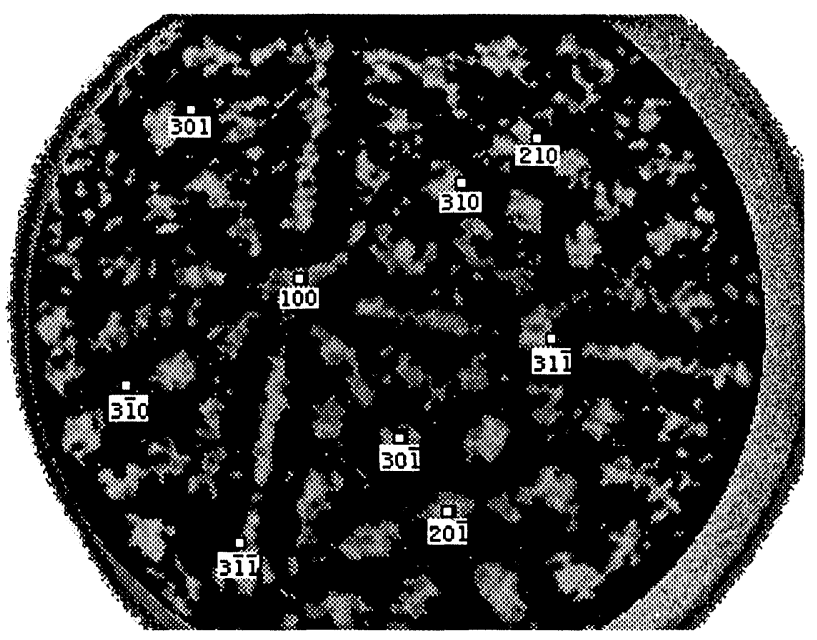

Figure 3d Stainless steel image indexed by the computer.

construction of the template set required approximately one and a half hours. The averaging of the $10 \times 10$ pixel blocks required eight seconds and the flattening of the brightness gradient required 32 seconds. Enhancing the zone axis-background contrast required nearly ten minutes. The large convolution appears to be the bottleneck in the procedure. This bottleneck may be reduced through optimization of the code or through faster hardware. It should be noted that this procedure lends itself well to parallel processing. However, the silicon pattern had a better signal to noise ratio than the stainless steel patterns. This indicates that a more promising possibility for increasing the speed of the process is to obtain higher quality images. This could be accomplished through more sophisticated image capturing hardware. Front end filtering and higher capacity frame averaging would lead to better images resulting in less image processing required for obtaining correct solutions. Another possibility for acquiring higher quality images is to capture many images and then create a background image by averaging all of the images together. This average background image could be subtracted from the images resulting in a higher signal to noise ratios and removal of the brightness gradients. However, this may not work for materials of very strong textures where many of the zone axes of each image may be in similar positions.

Another approach to strengthening the procedure would be to calculate the theoretical ranking in the brightness of the poles. The intensity of the bands in the BKD patterns is approximately proportional to the square of the structure factor. The structure factor, $F$, is given by the following equation (Sands, 1975) for a lattice plane $(h k l)$ :

$$
F(h k l)=\sum_{j} f_{j} e^{2 \pi i\left(h x_{j}+k y_{j}+l z_{j}\right)}
$$

where $(x y z)_{j}$ is the relative position of atom $j$ in the unit cell and $f_{j}$ is the atomic scattering factor. The atomic scattering factor is a function of the interatomic 
spacing, $d_{h k l}$, and can be found in the International Tables for X-ray Crystallography. Since the zone axes are formed by the intersection of bands; the relative intensity of a zone axis can be approximated by simply adding the squares of the structure factors together for the bands intersecting to form the zone axis. However, not all bands would need to be considered since many would have negligible intensities. Once a structure factor for each zone axis in the global set used for constructing the templates has been determined; the zone axes can be ranked according to their structure factors. The recognition procedure could then be modified to recognize this ranking by introducing a weighting term into Eq. (7) as follows:

$$
\bar{I}^{i}=\frac{1}{n^{i}} \sum_{j}^{n^{i}} w_{j}^{i} I\left(x_{j}^{i}, y_{j}^{i}\right)
$$

where $w_{j}^{i}$ would be a weighting factor proportional to the square of the structure factor for the $[h k l]$ corresponding to the $j$ th pole of template $i$.

\section{CONCLUSIONS}

These initiatory results show that automated indexing of the BKD patterns is realizable. However, for single orientation measurements to be effectively used in texture analysis, significant increases in the speed of the procedure would be required. This could be accomplished with more sophisticated image capture hardware and software and/or by incorporating more of the physics associated with BKD by increasing the importance of the higher intensity zone axes as discussed in the previous section. This should increase the discriminatory ability of the technique in recognizing the match of the pattern being analyzed with the correct template. This would help decrease the amount of image processing time required to determine the correct solution.

A test of the statistical reliability of the current procedure is needed. The results for a significant number of BKD patterns obtained by the automated approach would simply be compared to the results obtained by an operator. A statistical comparison could be made by comparing the ODFs for the two independent data sets.

\section{References}

Bunge, H. J. Texture Analysis in Materials Science. Butterworths, London (1982).

Coates, D. G. Phil. Mag. 16 (Series 8), 1179 (1967).

Dingley, D. J. Scanning Electron Microscopy II, 569 (1984).

Sands, D. E., Introduction to Crystallography. W. A. Benjamin, Inc., London (1975).

Schmidt, N.-H., and N. Ø. Olesen, Canadian Mineralogist 27, 15 (1989).

Venables, J. A. and C. J. Harland, Phil. Mag. 27, 1193 (1973).

Wright, S. I., and B. L. Adams, Textures and Microstructures, 12, 65-76 (1990). 